\title{
Satisfacción con el cuidado de Enfermería del adulto mayor hospitalizado
}

Rubi Contreras Nuñez*, Maribel Coronado Martínez*, Nora Hilda López Villanueva*, Dra. Rosa Ma. Aguilar Hernández**, M.S.P. Aurora Félix Alemán** * y M.C.E. Ma. Del Carmen Vega Alanís***.

* Tesistas de Licenciatura en Enfermería de la Unidad Académica Multidisciplinaria Matamoros-UAT (UAMM-UAT)., **Profesora de tiempo completo, Coordinadora de investigación, directora de tesis. UAMM-UAT, ***Profesoras de Tiempo Completo, co-directoras de tesis. UAMM-UAT

Los adultos mayores hospitalizados son un grupo vulnerable que requiere cuidado de Enfermería de calidad; esta conlleva satisfacción valorada a través de: relación Enfermera-paciente, comunicación efectiva, información oportuna y habilidad técnica profesional. Para identificar satisfacción de adultos mayores con cuidado de Enfermería se realizó un estudio descriptivo transversal con 45 adultos mayores hospitalizados en Medicina Interna y Cirugía de dos instituciones de salud. Mediante entrevista se aplicó una escala de Likert de 23 reactivos con cinco categorías: de completo acuerdo (5) a total desacuerdo (1). A mayor puntuación mayor satisfacción. Procesamiento y análisis con programa estadístico SPSS. Resultados: edad media 71 años, escolaridad media 6 años. Completo acuerdo: Enfermera agradable con el paciente (62.2\%), debería ser más atenta $(60.0 \%)$, se siente mejor al platicar con ella (75.6\%), le explica en lenguaje sencillo (73.3\%), brinda información sobre exámenes (68.9\%). Total desacuerdo: le molesta cuando la Enfermera le habla como si fuera niño (60.0\%). Alta puntuación (85\%) en evaluación general de satisfacción con el cuidado de Enfermería. Coincidente con estudios previos en que a mayor edad mayor satisfacción con el cuidado de Enfermería.

Palabras clave: Satisfacción con el cuidado, Cuidado de Enfermería, Adulto Mayor, Hospitalización

\section{Albstract}

Older adults hospitalized are a vulnerable group that require quality nursing care; this imply satisfaction valued through patient-nursing relationship, effective communication and information and professional technical competence. In order to identify older adults' satisfaction with nursing care it was carried out a transactional descriptive study with 45 hospitalized older adults in medical and surgical wards of two health institutions. Through interview it was applied a Likert scale of 23 items and five categories: complete agree (5) to total disagree (1). Instrument was translated and validated, it was obtained Cronbach's alpha coefficient from 78 (12). Results. Age mean 71, years of school mean 6. Complete agree: nursing pleasant with patient (62.2\%), she should be more attentive with patient (60.0\%), the patient feels better when nurse talks with him(75.6\%), nurse explains to patient in sample language (73.3\%), nurse gives information about exams (68.9\%). Total disagree: Patient gets angry when nurse talks him like a child (60.0\%). High score (85\%) in general evaluation about satisfaction with nursing care. This study is coincident with previous studies about to higher age higher satisfaction with nursing care.

Key words: Satisfaction with nursing care, Nursing care, Older adult, Hospitalized 


\section{INTRODUCCIÓN}

Los adultos mayores son de interés para la profesión de Enfermería, por el aumento en la expectativa de vida y consecuente incremento de la población en este grupo de edad que obliga a poner atención a sus necesidades. Los cambios degenerativos de la edad avanzada, biológicos, psicológicos y sociales ${ }^{1}$ exponen a los adultos mayores a riesgo a la salud, que de no atenderse oportunamente, conllevan enfermedades que los conduce a la hospitalización, lo que constituye un factor de riesgo agregado. La fragilidad y el contacto con personas de diferentes patologías pueden prolongar la estancia del adulto mayor por complicaciones que no se resuelven fácilmente. Además, pueden producirse cuadros agudos graves y conducir a la muerte. El profesional de Enfermería tiene un papel relevante en la atención de los pacientes hospitalizados; ya que los atiende las 24 horas del día y porque su actividad profesional se centra en la atención a las necesidades y los problemas reales y potenciales derivados de su proceso patológico. Los conocimientos teóricos, la experiencia práctica y la sensibilidad de las Enfermeras son el fundamento para identificar necesidades y problemas en los pacientes hospitalizados. El cuidado de la salud es la esencia de la profesión de Enfermería. Cuidar implica establecer una relación de ayuda con el paciente y su familia, para lo cual es necesaria la relación estrecha y comunicación efectiva. Esto requiere de recursos intelectuales y de intuición para la toma de decisiones al realizar acciones o actividades que responden a las necesidades particulares de los pacientes. Algunos autores ${ }^{2}$ han definido la satisfacción del paciente como la congruencia entre las expectativas de calidad del cuidado que los pacientes esperan y la que obtienen. El cuidado de Enfermería en los adultos mayores tiene una mayor relevancia, en virtud de que presentan mayores necesidades y precisan una atención más estrecha, por lo que la satisfacción de estos pacientes se obtiene como resultado de un trato humano, individualizado y afable, que los hacen sentir personas únicas en la medida en que se atienden sus necesidades particulares y se logra su bienestar. La calidad del cuidado de Enfermería se conoce por la satisfacción con dichos cuidados que los pacientes manifiestan. Las Enfermeras podrán proporcionar un cuidado de Enfermería de calidad si cuentan con los conocimientos teóricos y las habilidades técnicas y humanísticas; pero además, que brinden la confianza que favorezca la comunicación para establecer un dialogo abierto con el paciente para que exprese sus dudas, temores e inquietudes. Los pacientes adultos mayores, por sus condiciones de deterioro físico, mental y social, son un grupo vulnerable que requiere mayor atención y cuidado por parte de la familia y de los profesionales de Enfermería, por lo que es posible que por su gran necesidad de cuidado den mayor valor al cuidado de Enfermería. El estudio sobre la revisión de literatura realizada por Johansson y cols. ${ }^{3}$ con 30 reportes de investigación sobre satisfacción del paciente realizado en tres países desarrollados reporta que los pacientes de mayor edad tienden a estar más satisfechos con el cuidado de Enfermería. Maldonado y cols. ${ }^{4}$ realizaron un estudio con adultos mayores en un contexto comunitario y encontraron que el sexo y la edad influyen en la satisfacción con el cuidado. A mayor edad del usuario mayor satisfacción con el cuidado. Los hombres de 70 a 75 años se mostraron satisfechos con el cuidado en un 100\%.

La mayoría de los estudios analizados sobre satisfacción del paciente con el cuidado de Enfermería fueron realizados en entornos hospitalarios con pacientes adultos. Estos estudios fueron realizados en México ${ }^{5}$ 6, 7,8, en Venezuela ${ }^{9}$, en Jordania ${ }^{10}$ y en Finlandia ${ }^{11}$. Gutiérrez y cols. ${ }^{5}$ encontraron que las Enfermeras no respetan la intimidad de los pacientes (51\%), no se dirigen a los pacientes por su nombre o apellido (32\%), los pacientes no conocen el nombre de la Enfermera que los atiende (49\%) y más de la mitad de los pacientes (58\%) refiere la atención de Enfermería como buena. Castañeda y Cols. ${ }^{6}$ encontraron una media de satisfacción de 91.51 y mediana de 93, lo que muestra satisfacción del usuario con el cuidado de Enfermería. Los usuarios del sexo masculino presentaron un nivel de satisfacción más alto (93.5). En el estudio de Ponce y Cols. ${ }^{7}$, para la mitad de los pacientes la calidad de atención de Enfermería fue insuficiente. En el estudio de Balseiro y cols. ${ }^{8}$ los pacientes consideraron que reciben un trato de Enfermería con calidez y amabilidad (80\%), que la relación Enfermera paciente es excelente (85\%) y que las Enfermeras desarrollaron de manera excelente sus procedimientos (88\%). Las autoras concluyen que los usuarios calificaron de buena a excelente tanto la calidad de la atención proporcionada por las Enfermeras como la satisfacción que obtuvieron por la atención recibida.

El estudio de Caligiore y Díaz ${ }^{9}$ reporta que los pacientes consideran el trato del personal de Enfermería como bueno (82\%); así como que son atendidos en forma satisfactoria (78\%) y oportuna (54\%). El estudio de Mrayyan ${ }^{10}$, comprendió tres aspectos: satisfacción del trabajo de las Enfermeras, satisfacción de los pacientes y calidad del cuidado de Enfermería. Respecto a la satisfacción de los pacientes con el cuidado de Enfermería, el resultado fue moderadamente satisfecho. Las autoras concluyen que existe interrelación de la satisfacción de las Enfermeras con el trabajo, la calidad del cuidado de Enfermería y la 
satisfacción de los pacientes. Suhonen y cols. ${ }^{11}$ estudiaron en pacientes quirúrgicos la asociación entre cuidado individualizado, satisfacción del paciente con el cuidado de Enfermería y la calidad de vida relacionada con la salud. Los resultados sobre satisfacción del paciente fue: muy satisfactorio en apoyo individualizado mediante actividades específicas de Enfermería; más satisfecho en atención a las necesidades de cuidado técnico científico y menos satisfecho con interés a sus necesidades de información.

El presente estudio se realizó para responder a la pregunta de investigación iCuál es el nivel de satisfacción con el cuidado de Enfermería del adulto mayor hospitalizado en los servicios de Medicina Interna y Cirugía General?

\section{METODOLOGÍA}

El diseño fue descriptivo-transversal, se encuestaron 45 adultos mayores hospitalizados en medicina interna y cirugía general de dos instituciones de segundo nivel de atención. Para medir la Satisfacción del Paciente con el Cuidado de Enfermería se utilizó un instrumento diseñado por Hinshaw y Atwood en 1982 denominado PSI (Patient Satisfaction Instrument), el cual fue traducido, adaptado y validado por Acevedo y cols. ${ }^{12}$ Validación del instrumento con un Alpha de Cronbach de .78. El instrumento de escala tipo Likert con 23 reactivos comprende tres dimensiones: Cuidado Técnico Profesional, Relación Interpersonal Enfermera-Paciente y Educación al Paciente. Las opciones de respuesta fueron: Completamente de Acuerdo, Moderado Acuerdo, Mínimo Acuerdo, en Desacuerdo y Totalmente en Desacuerdo. La escala de puntuación mínima 23 y máxima 115. La puntuación arriba de 75 se considera que el paciente está satisfecho con el cuidado otorgado por Enfermería

Se capturó la información en el Programa Estadístico para las Ciencias Sociales (SPSS) versión 15.0. Se utilizaron las estadísticas descriptivas para el análisis de los datos. Se cumplió con lo dispuesto en el Reglamento de la Ley General de Salud en Materia de Investigación para la Salud. El estudio se consideró de riesgo mínimo. Se mantuvo el anonimato, la confidencialidad de la información y su derecho de retirar su participación si así lo decidían. Una vez aceptadas las condiciones se firmó el consentimiento informado por parte del entrevistador y el entrevistado ${ }^{13}$.

\section{RESULTADOS}

Los adultos mayores estaban hospitalizados en el Hospital General de la Secretaría de Salud (62.2\%) y en el Hospital del Instituto Mexicano del Seguro Social (37.8\%); en los servicios de cirugía (57.8\%) y Medicina Interna (42.2\%) (Tabla 1). La media de edad fue de 71 años, con predominio del sexo masculino (53.3\%). La media de escolaridad fue de 5.2 años. Menos de la mitad casados (44.4\%) y no trabajan (42.2\%). En la mayoría de los participantes (62.2\%) la hospitalización fue subsecuente, con una media de estancia hospitalaria de 4.2 (Tabla 2). En la dimensión de Cuidado Técnico Profesional, la satisfacción de los adultos mayores con el cuidado de Enfermería; los reactivos con predomi-

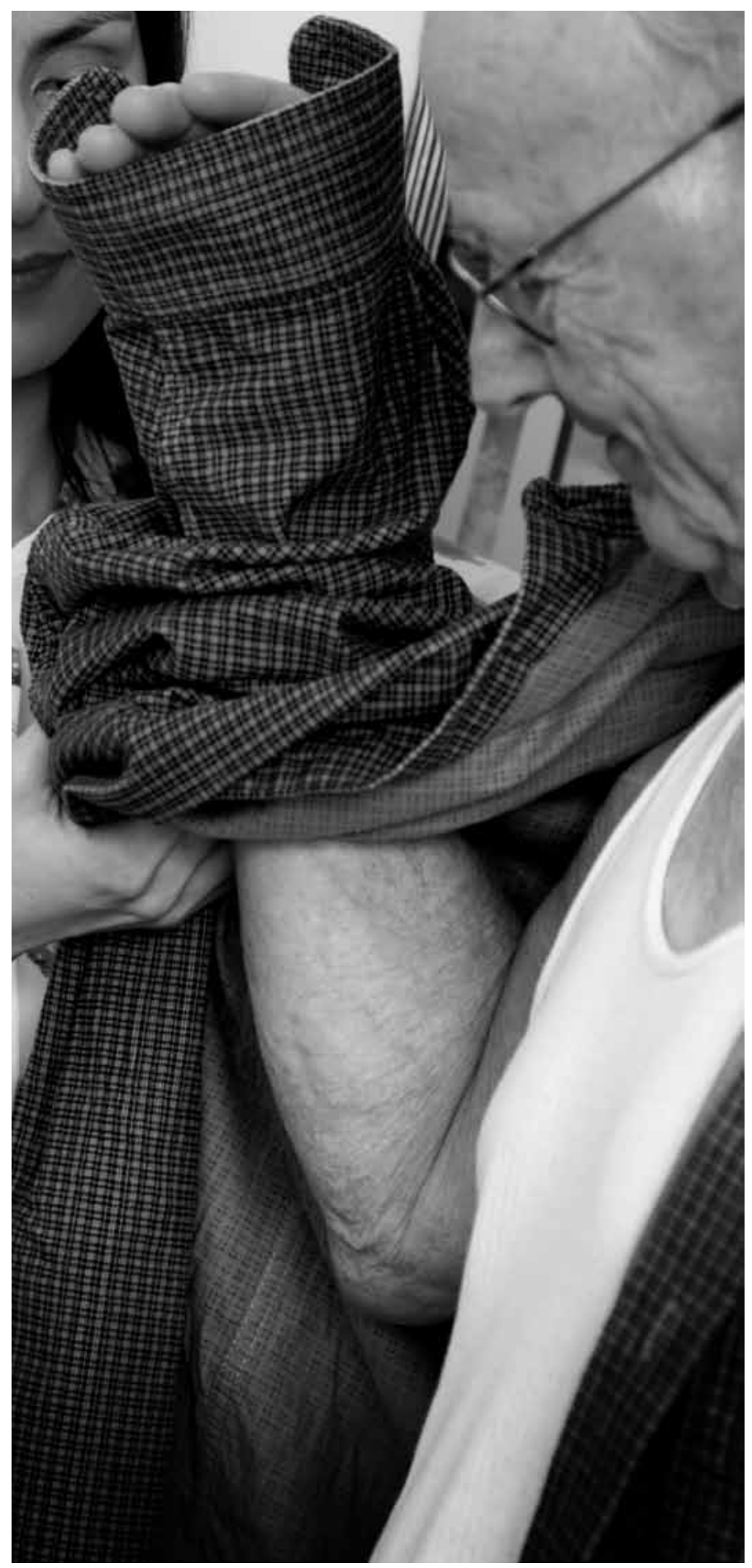


nio en Completo Acuerdo fueron: la Enfermera le enseña a llevar a cabo indicaciones médicas (48.9\%); se acerca a los pacientes para brindarle consejos (44.4\%), conoce sobre el cuidado que le brinda (46.7\%) y muestra habilidad para realizar los procedimientos (44.4\%). Los reactivos con predominio de Total Desacuerdo fueron: la Enfermera es desordenada y se altera en su trabajo (40.0\%) y no es precisa en su trabajo (40.0\%) (Tabla 3 ).

En la dimensión Relación Interpersonal Enfermera Paciente, los reactivos con predominio en Completo Acuerdo fueron: El paciente se siente mejor al platicar con la Enfermera (75.6\%), la Enfermera se muestra agradable cuando está con el paciente (62.2\%), la Enfermera debería ser mas atenta de lo que es (60.0\%) y el paciente se siente con libertad para preguntarle a la Enfermera cuestiones de salud (55.6\%).

Sólo un reactivo predominó en Total Desacuerdo: le molesta cuando la Enfermera le habla como si fuera niño (60.0\%) (Tabla 4). En la dimensión Educación al Paciente, los reactivos con predominio en Completo Acuerdo fueron: la Enfermera le explica en lenguaje sencillo (73.3\%), quisiera que la Enfermera le brindara información de los resultados de exámenes (68.9\%) y considera que la Enfermera le da suficientes explicaciones sobre las razones de los exámenes que se le solicitan (64.4\%) (Tabla 5). La evaluación general de la Satisfacción con el Cuidado de Enfermería del Adulto Mayor obtuvo mayor puntuación en el rango de 80 a 89 puntos que corresponde a la categoría de Moderado Acuerdo, con el $84.0 \%$ de los participantes (Tabla 6).

\section{DISCUSIÓN}

La satisfacción con el cuidado de Enfermería de los adultos mayores hospitalizados fue evaluada en sus tres dimensiones: Cuidado Técnico Profesional, Relación Interpersonal Enfermera-Paciente y Educación al Paciente. Esta forma de evaluación es coincidente con los 30 reportes de investigación analizados por Johansson y cols. ${ }^{3}$, y con los estudios de Castañeda y cols. ${ }^{6}$ y de Suhonen ${ }^{11}$. En cuanto a la dimensión de Cuidado Técnico Profesional, poco menos de la mitad de los adultos mayores estuvo de acuerdo en que Enfermera conoce sobre el cuidado que le brinda y que muestra habilidad para realizar los procedimientos; por su parte Suhonen y cols. ${ }^{11}$, mencionan que los pacientes en su estudio se mostraron más satisfechos con la Atención a las Necesidades de Cuidado Técnico Científico. Esta diferencia puede deberse a que los procedimientos que realizan las Enfermeras quirúrgicas son muy específicos y los pa- cientes tienen oportunidad de apreciar sus habilidades. También, Balseiro y Cols. ${ }^{8}$ encontraron que, en opinión de los pacientes, las Enfermeras desarrollaron de manera excelente sus procedimientos. En la dimensión Relación Interpersonal Enfermera-Paciente se encontró que una gran proporción de adultos mayores consideran que la Enfermera se muestra agradable; sin embargo, también opinaron que debería ser más amable. Estos datos son coincidentes con los de Caligiore y Díaz ${ }^{9}$ quienes encontraron que los pacientes consideran como bueno el trato que les brindan las Enfermeras. El estudio de Balseiro y Cols. ${ }^{8}$ revela que los pacientes consideran que la relación Enfermera paciente es excelente.

En la dimensión Educación al Paciente, los adultos consideran que las Enfermeras brindan explicación en cuanto a exámenes y utilizan un lenguaje sencillo. Por el contrario, Suhonen y cols. ${ }^{11}$ encontraron que los pacientes se mostraron menos satisfechos con el interés a sus necesidades de información.

En este estudio se evidencia que los adultos mayores hospitalizados muestran alta satisfacción con el cuidado de Enfermería, similar a lo encontrado por Castañeda y cols. ${ }^{6}$, Maldonado y cols. ${ }^{4}$ y Balseriro y cols. ${ }^{8}$. Por el contrario, Ponce y cols. ${ }^{7}$ encontraron que los pacientes refirieron la atención de Enfermería como insuficiente.

\section{CONCLUSIONES}

La mejor manera de evaluar el trabajo de Enfermería es la satisfacción que los pacientes tienen en cuanto al cuidado que reciben. Esta satisfacción se obtiene en la medida en que los pacientes reciben un trato de Enfermería de calidad y sienten que sus necesidades son cubiertas. El estudio evidencia que los adultos mayores muestran alta satisfacción con el cuidado de Enfermería. Sin embargo, opinaron que las Enfermeras deberían ser más atentas y no les molesta que ellas les hablen como a un niño.

\section{RECOMENDACIONES}

En consideración a que el cuidado de Enfermería es la esencia de la profesión, es necesario realizar estudios sobre el cuidado que se brinda; en particular con en grupo de población que mayor atención requiere: los adultos mayores. Es necesario generar conocimiento a partir de la experiencia de los receptores del cuidado, conocimiento que sea útil para planear mejor las acciones de Enfermería; y de esta manera, lograr la eficiencia en el cuidado de Enfermería en este vulnerable grupo de población. 
TABLA 1

Instituciones y Servicios de Estancia del AdultoMayor Hospitalizado

\begin{tabular}{lllllll}
\hline $\begin{array}{l}\text { Instituciones } \\
\text { Servicios }\end{array}$ & \multicolumn{2}{l}{ Hospital General } & IMSS & \multicolumn{2}{c}{ Total } \\
\hline Medicina Interna & $f$ & $\%$ & $f$ & $\%$ & $f$ & $\%$ \\
\hline Cirugía & 12 & 26.7 & 7 & 15.5 & 19 & 42.2 \\
\hline Total & 16 & 35.5 & 10 & 22.3 & 26 & 57.8 \\
\hline
\end{tabular}

Fuente: Instrumento Satisfacción del Paciente $n=45$

TABLA 2

Características Sociodemográficas Relevantes de los Adultos Mayores Hospitalizados

\begin{tabular}{lccccc}
\hline Variable & Categoría & $\mathbf{f}$ & \% & Media & Mediana \\
\hline Edad & 65 & 5 & 11.1 & 71 & 71 \\
Sexo & Masculino & 24 & 53.3 & - & - \\
Escolaridad & 6 & 9 & 20 & 5.2 & 6 \\
Estado civil & Casado & 20 & 44.4 & - & - \\
Ocupación & No trabaja & 19 & 42.2 & - & - \\
Días estancia & 4 & 19 & 42.2 & 4.8 & 4 \\
Hospitalización & Subsecuente & 28 & 62.2 & 1.6 & 2 \\
\hline
\end{tabular}

Fuente: Instrumento Satisfacción del Paciente. $n=45$

TABLA 3

Satisfacción con el Cuidado de Enfermería del Adulto Mayor Hospitalizado en la dimensión Cuidado Técnico Profesional

\begin{tabular}{lcccc}
\hline Cuidado Técnico & \multicolumn{2}{c}{ Completo acuerdo } & \multicolumn{2}{c}{ Total desacuerdo } \\
Profesional & $\mathbf{f}$ & $\%$ & $\mathbf{f}$ & $\%$ \\
\hline $\begin{array}{l}\text { La Enfermera le enseña a llevar } \\
\begin{array}{l}\text { Labo indicaciones médicas } \\
\text { y se altera en su trabajo }\end{array}\end{array}$ & 22 & 48.9 & 4 & 8.9 \\
$\begin{array}{l}\text { Se acerca a los pacientes para brindar } \\
\text { consejos. }\end{array}$ & 3 & 6.7 & 18 & 40.0 \\
$\begin{array}{l}\text { Conoce sobre el cuidado } \\
\text { que le brinda }\end{array}$ & 20 & 44.4 & 1 & 2.2 \\
$\begin{array}{l}\text { Es lenta para dar cuidados } \\
\text { La Enfermera no es precisa } \\
\text { en su trabajo }\end{array}$ & 21 & 46.7 & 1 & 2.2 \\
$\begin{array}{l}\text { Muestra habilidad para realizar } \\
\text { procedimientos }\end{array}$ & 2 & 4.4 & 10 & 22.2 \\
\hline
\end{tabular}

Fuente: Instrumento Satisfacción del Paciente. $n=45$ 
TABLA 4

Satisfacción con el Cuidado de Enfermería del Adulto Mayor Hospitalizado en la Dimensión Relación Interpersonal Enfermera Paciente

\begin{tabular}{|c|c|c|c|c|}
\hline \multirow[t]{2}{*}{ Relación interpersonal Enfermera-paciente } & \multicolumn{2}{|c|}{$\begin{array}{l}\text { Completamente de } \\
\text { acuerdo. }\end{array}$} & \multicolumn{2}{|c|}{$\begin{array}{c}\text { Totalmente en } \\
\text { desacuerdo. }\end{array}$} \\
\hline & $f$ & $\%$ & $f$ & $\%$ \\
\hline Debería ser mas amable & 21 & 46.7 & 2 & 4.4 \\
\hline $\begin{array}{l}\text { La Enfermera se muestra agradable cuando } \\
\text { está con el paciente }\end{array}$ & 28 & 62.2 & 1 & 2.2 \\
\hline $\begin{array}{l}\text { El paciente se siente con libertad para preguntarle } \\
\text { a la Enfermera cuestiones de salud }\end{array}$ & 25 & 55.6 & 1 & 2.2 \\
\hline La Enfermera debería ser más atenta de lo que es & 27 & 60.0 & 1 & 2.2 \\
\hline Lo comprende en su sentir & 21 & 46.7 & 5 & 11.1 \\
\hline Acude a ella en sus problemas & 21 & 46.7 & 3 & 6.7 \\
\hline Tiene tiempo para platicar con usted. & 9 & 20.0 & 6 & 13.3 \\
\hline Se muestra comprensiva y lo escucha & 20 & 44.4 & 2 & 4.4 \\
\hline No muestra paciencia & 5 & 11.1 & 17 & 37.8 \\
\hline $\begin{array}{l}\text { Le molesta cuando la Enfermera le habla } \\
\text { como si fuera un niño }\end{array}$ & 7 & 15.6 & 27 & 60.0 \\
\hline Se siente mejor al platicar con la Enfermera & 34 & 75.6 & _- & _ \\
\hline
\end{tabular}

Fuente: Instrumento Satisfacción del Paciente $n=45$

\section{TABLA 5}

Satisfacción con el Cuidado de Enfermería del Adulto Mayor Hospitalizado en la Dimensión Educación al Paciente.

\begin{tabular}{|c|c|c|c|c|}
\hline \multirow[t]{2}{*}{ Educación al Paciente } & \multicolumn{2}{|c|}{$\begin{array}{c}\text { Completamente de } \\
\text { acuerdo }\end{array}$} & \multicolumn{2}{|c|}{$\begin{array}{c}\text { Totalmente en } \\
\text { desacuerdo. }\end{array}$} \\
\hline & f & $\%$ & $f$ & $\%$ \\
\hline La Enfermera le explica en lenguaje sencillo & 33 & 73.3 & - & - \\
\hline $\begin{array}{l}\text { Quisiera que la Enfermera le brindará información de los resultados } \\
\text { de exámenes }\end{array}$ & 31 & 68.9 & 2 & 4.4 \\
\hline Es fácil de entender lo que la Enfermera le explica & 21 & 46.7 & 1 & 2.2 \\
\hline Da instrucciones precisas & 21 & 46.7 & - & - \\
\hline $\begin{array}{l}\text { Considera que la Enfermera le da suficientes explicaciones sobre las } \\
\text { razones de exámenes que se le solicitan }\end{array}$ & 29 & 64.4 & 4 & 8.9 \\
\hline
\end{tabular}

Fuente: Instrumento Satisfacción del Paciente $n=45$ 
TABLA 6

Satisfacción con el Cuidado de Enfermería del Adulto Mayor Hospitalizado

\title{
Satisfacción
}

\author{
Categoría de respuesta
}

5 Completamente de acuerdo ( 99 a 118 pts)

4 Moderado acuerdo (80 a 98 pts.)

3 Mínimo acuerdo ( 61 a 79 pts.)

2 En desacuerdo ( 42 a 60 pts.)

1 Totalmente en desacuerdo ( 23 a 41 pts.) $f$

4

9.4

45

$\%$

38

84.0

3

6.6

100

Total

Fuente: Instrumento Satisfacción del Paciente $n=45$

\section{REFERENCIAS BIBLIOGRÁFICAS}

1 Hayflick, L. Cómo y porque envejecemos. Hender: Barcelona, 2001. p. 49-51.

2 Mrayyan, M.T. Jordanian nurses'satisfaction and quality of nursing care. International Nursing Review. 2006, vol. 53, 224-230.

3 Johansson, P., Oleni, M. y Fridlung, B. Patient satisfaction with nursing care in the context of health care: a literature study. Nordic Collage of Caring Science, Scand j Caring Sci. 2002, Vol. 16; 337-344.

4 Maldonado, G., Medrano, M.R., Mendiola, S.V., Nieto, M.J. y Flores, G. El cuidado del adulto mayor. Desarrollo científico. Enfermería. 2005, Vol.13, 169-189.

5 Gutiérrez S, Mederos L, Vásquez E J, Velásquez M. Derechos del paciente hospitalizado: Responsabilidad en la práctica de Enfermería. Rev Enferm IMSS 2001, Vol. 9, 15-18.

6 Castañeda M H, Acevedo G I, Aguilera A, González F, Rangel S, Acevedo G, Garza R, Meléndez M C, Pineda M, Patiño M L. Relación de las características sociodemográficas de los usuarios y su nivel de satisfacción con la atención de Enfermería. Facultad de Enfermería Tampico Tamaulipas. 2005, 23-29.

7 Ponce J, Reyes H, Ponce G. Satisfacción laboral y calidad de atención de Enfermería en una unidad médica de alta especialidad. Rev Enferm IMSS 2006, Vol 14,65-73.

8 Balseiro L, Martínez P, Ángeles R, Cortés A, De la Rosa M, Flores A, Flores P P. La calidad de la atención de Enfermería a los pa- cientes de un hospital regional del ISSSTE, en México. D. F. Un estudio basado en el grado de satisfacción de los usuarios. Revista Universitaria ENEO- UNAM 2007, Vol.4,.9-13.

9 Caligiore I. y Díaz J A. Satisfacción del usuario del área de hospitalización de un hospital de tercer nivel. Mérida, Venezuela. Revista electrónica semestral de Enfermería. Enfermería Global 2003 Número 2,1.

10 Mrayyan M T. Jordanian nurses'satisfaction and quality of nursing care. International Nursing Review 2006 Vol. 53, 224-230.

11 Suhonen R, Valimaki M, Leino-Kilpi H. Individualized care, quality of life and satisfaction with nursing care. Journal of Advanced Nursing 2005, Vol. 50,283-292.

12 Acevedo G, Castañeda H, Garza R, González F, Meléndez C, Acevedo G. Adaptación de un Instrumento para Medir la Satisfacción del Paciente con el Cuidado de Enfermería. Desarrollo Científico de Enfermería 2006, Vol. 14, 245-250.

13 Poder Ejecutivo Federal. Reglamento de la Ley General de Salud, en materia de investigación para la salud. Recuperado el 23 de mayo de 2007. Disponible en: http://hgm.salud.gob. mx/pdf/marco_juridico/reglamentos/regla_05.pdf

\section{DIRECCIÓN PARA CORRESPONDENCIA}

Dra. Rosa Ma. Aguilar Hernández: raguilar@uat.edu.mx 